\title{
SEMANTIC TAGGING OF REQUIREMENT DESCRIPTIONS: A TRANSFORMER-BASED APPROACH
}

\author{
Joschka Kersting ${ }^{1}$ and Frederik S. Bäumer ${ }^{2}$ \\ ${ }^{1}$ Paderborn University, Warburger Str. 100, 33098 Paderborn, Germany \\ ${ }^{2}$ Bielefeld University of Applied Sciences, Interaktion 1, 33619 Bielefeld, Germany
}

\begin{abstract}
The idea of On-The-Fly Computing is to compose and provide software services ad hoc to end users. This is a highly automated process, which has to start with requirement descriptions in natural language. The main reason is that end users prefer natural language software requirements over formal language due to the usability and accessibility. However, there are good reasons for not using natural language, such as the susceptibility to errors. Nevertheless, to enable processing, a machine interpretation of these initial natural language text data is of fundamental importance. In this short paper, we present our ongoing work on $\mathrm{REaCT}^{2}$, a tool that is able to process requirement documents regarding semantic roles. This information can be reliably extracted and used to provide software services. More specifically, $\mathrm{REaCT}^{2}$ achieves an F1 Score of $90 \%$ for the semantic tagging of requirements and outperforms existing software solutions in this task.
\end{abstract}

\section{KEYWORDS}

Software Requirements, Natural Language Processing, Transfer Learning, On-The-Fly Computing

\section{INTRODUCTION}

The idea of On-The-Fly (OTF) Computing employs individual software requirements in natural language (NL) provided by end users for an automatic composition of individual software services (Karl et al. 2019: 1-2). Simply put, OTF Computing aims at providing tailored software services to individuals. Here, a way must be found to provide information on what end users need: It is neither expected that users are domain experts, nor is knowledge about formal requirement description languages common among average Internet and smartphone users. Thus, requirements are given in NL, which is challenging (cf. Figure 1). The requirements must be extracted very quickly and reliably as they are the only source of information for the software composition while the whole system is expected to work OTF. However, finding important and useful information in NL service descriptions is a challenging task because they are unstructured and contain off-topic information. Apart from that, canonical core functionalities such as "role", "priority" or "action" (cf. Fig. 1) are neither provided in a certain order, nor easy to extract. However, relations among requirements are important for figuring out which requirements determine others. This determines the functionalities of an application and thus the functionalities must be extracted with respect to their contents and dependencies.

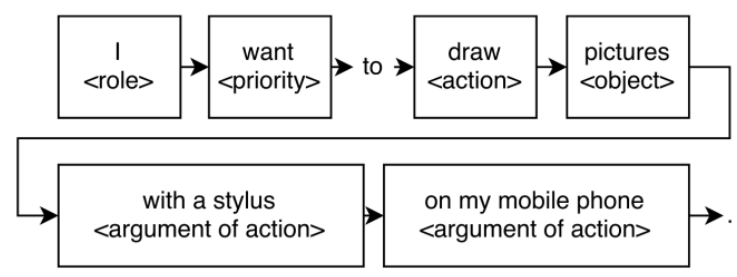

Figure 1. Annotated requirement sample (Dollmann/Geierhos 2016: 1810) 
Up to now, there is little research done on processing these software requirements in the OTF Computing context. For example, there is a tool named REaCT (Requirements Extraction and Classification Tool), which uses machine learning (ML) for information extraction (Dollmann/Geierhos 2016: 1811ff.). It works on textual requirement descriptions and tries to transfer the most important entities for functional requirements. From the technical side, this involves dividing description texts into sentences and classifying them into off- and on-topic components. After this step, attribute-value pairs are extracted from the on-topic sentences containing functional requirements in order to iteratively fill a predefined template, starting with the most important elements such as subjects, actions, predicates and objects (e.g. indirect objects). Since $\mathrm{REaCT}$ works very slowly due to extensive preprocessing and not very reliably - mainly due to the fact that there is hardly any training data for the chosen learning methods - a more reliable, ML-based approach was published called $\mathrm{REaCT}_{\text {frame }}$ (Kim et al. 2017), which, however, also suffers from the fact that there is hardly any training data.

Next to this, the idea of extracting requirements without ML but rule-based is raised (Caron et al. 2018). Due to the predefined sets of rules, these approaches are very maintenance-intensive. Other approaches focus on high-quality requirements or assume getting high-quality texts, which makes such tools unsuitable for the OTF Computing domain. However, most existing approaches are developed for experts rather than for end users or an application in everyday life (Dollmann/Geierhos 2016: 1808). Consequently, we continue the work of Dollmann \& Geierhos (2016) and Kim et al. (2017) and improve their approaches.

In this paper we present our work on $\mathrm{REaCT}^{2}$, which is able to perform high-quality semantic annotations in requirement descriptions and thus provides a solid basis for OTF software composition.

\section{REACT ${ }^{2}$ : SEMANTIC TAGGING WITH TRANSFORMERS}

The domain of OTF Computing, like many tasks in Natural Language Processing (NLP), suffers from a lack of proper data that may be used for training ML and especially data-driven deep learning models. REaCT and $\mathrm{REaCT}_{\text {frame }}$ were trained with a small but manually annotated dataset (Dollmann/Geierhos 2016: 1810; Kim et al. 2017: 3f.) and the annotation of new datasets cannot be conducted, as manual labor is cost-intensive and not feasible. There are few methods that can work with these tiny data amounts. However, current breakthroughs in NLP research enable researchers to incorporate general knowledge from large, unannotated text data into models that help ML tasks with their semantic understanding of the data. In the following, we use this to make the extraction of requirements faster and more robust.

\subsection{Background}

Recent developments in the domain of NLP are led by transformers (Vaswani et al. 2017: 5998ff.): a deep learning architecture solely based on attention mechanisms that can efficiently process large amounts of text data to produce a semantic understanding from it, which is incorporated into a language model. These language models can be fine-tuned on decisive tasks such as Named Entity Recognition (NER), where entities such as places or organizations are extracted from texts (Devlin et al. 2018). Moving from using word vectors in downstream models to constructing language models and fine-tune them for tasks was a shift in NLP methodology and led to large improvements over previous approaches. The advantage lies in the use of information incorporated in all layers of a model rather than only the last layer (Peters et al. 2018: 2227ff.).

BERT (Devlin et al. 2018) and RoBERTa (Liu et al. 2019) are examples of transformers that are trained on large data amounts such as newspaper and encyclopedia articles. As an example, BERT was trained on a book corpus and Wikipedia articles, RoBERTa on a broader text corpus including news articles and web pages. The authors argue that this can be considered favorable over sentence data because BERT as a transformer is able to learn from long sequences and reason from context (Devlin et al. 2018; Liu et al. 2019). The main advantage of BERT is that it works bidirectionally and thus considers a whole sentence at once and not, like other models, in one or two directions (Devlin et al. 2018). Furthermore, Devlin et al. (2018) write that this feature allows them to obtain good evaluation scores in all tasks across the NLP domain. Consequently, many scholars developed follow-up works. Among the best, i.e. efficient and robust, is RoBERTa (Liu et al. 2019), but also BART (Lewis et al. 2019). BART also builds on GPT (Radford et al. 2018: 1ff.), another recent language model that has follow-up works, but is unidirectional. In contrast, the 
training of models such as BERT is conducted by masking words in a sentence and letting the language model guess the masked word using just the position in the sentence and the context words. In this manner, the model learns to embed the words in a vector space (Devlin et al. 2018) and thus words such as 'bank' receive different vectors based on the context, e.g. in relation to financial services and a sandbank in the sea. This is a useful trait for learning models which we employ for semantic analyses of NL requirement descriptions in On-the-Fly Computing.

\subsection{Approach}

In this short paper, we tested the recent transformer models of BERT and RoBERTa for our use case. That said, we fine-tuned pre-trained versions of both models by treating our use case as a tagging task. Here, every word received a tag corresponding to the current class, such as "action", "condition" or "component" (cf. Dollmann/Geierhos 2016: 1810; Kim et al. 2017: 5). We fine-tuned the models to tag whole sentences at once. An issue to deal with was the low number of some of the tags, which hindered a good performance that otherwise could have been realized with only few training epochs. Nevertheless, in contrast to Dollmann and Geierhos (2016) and Kim et al. (2017), we focus on the tagging part and, in the scope of this short paper, do not deal with sentence-based on- and off-topic classification. Furthermore, they invest heavily in preprocessing and feature engineering, a task prone to errors and mistakes that is obsolete when using transformer-based models. For our research, we used the training and test data of Dollmann and Geierhos (2016: 1809ff.) as well as the Simpletransformers deep learning software library (Rajapakse 2020), making it easy to download and fine-tune common transformer models. Having tested BERT in its smaller and larger variant for the English language and RoBERTa, also in its smaller and larger variant, it evolved that the large RoBERTa performed best in its original implementations (Devlin et al. 2018; Liu et al. 2019). While the results were good from beginning, we tuned the available parameters such as the maximum word length (padding), number of epochs, or batch size.

\section{EVALUATION AND DISCUSSION}

We evaluated our early work on $\mathrm{REaCT}^{2}$ on the basis of two criteria that are both very important for the OTF Computing use case: performance and accuracy ( $\mathrm{F} 1$ score). From the performance view, $\mathrm{REaCT}^{2}$ is four times as fast as $\mathrm{REaCT}$ in the original version and twice as performant as $\mathrm{REaCT}_{\text {frame }}$. This is a feature that directly contributes to end user benefit, as resulting software services are presented much faster to the end users. To be fair, it must also be said that training the models in $\mathrm{REaCT}^{2}$ takes longer than with the predecessors, which is, however, of no importance in our use case as long as the user experience remains unaffected.

Table 1. REaCT and REaCT $\mathrm{R}_{\text {rame }}$ vs. the proposed model (F1 scores)

\begin{tabular}{lrrr}
\hline Categories & REaCT (2016) & REaCT $_{\text {frame }}(\mathbf{2 0 1 7})$ & Proposed REaCT $^{\mathbf{2}} \mathbf{( 2 0 2 0 )}$ \\
\hline Action & 0.80 & 0.85 & $\mathbf{0 . 9 7}$ \\
Argument of action & 0.54 & $\mathbf{0 . 5 8}$ & 0.57 \\
Argument of sub-action & 0.31 & 0.28 & $\mathbf{0 . 8 5}$ \\
Component & 0.73 & $\mathbf{0 . 8 3}$ & 0.78 \\
Condition & 0.72 & $\mathbf{0 . 7 3}$ & 0.57 \\
Motivation & 0.40 & 0.75 & $\mathbf{0 . 8 4}$ \\
Object & 0.68 & 0.82 & $\mathbf{0 . 8 6}$ \\
Priority & $\mathbf{0 . 9 6}$ & 0.94 & 0.93 \\
Refinement of component & 0.15 & 0.36 & $\mathbf{0 . 9 5}$ \\
Refinement of object & 0.59 & 0.51 & $\mathbf{0 . 6 7}$ \\
Refinement of sub-object & 0.44 & $\mathbf{0 . 4 9}$ & 0.48 \\
Role & 0.89 & $\mathbf{0 . 9 6}$ & $\mathbf{0 . 9 6}$ \\
Sub-action & 0.45 & 0.59 & $\mathbf{0 . 9 3}$ \\
Sub-object & 0.34 & 0.50 & $\mathbf{0 . 9 2}$ \\
Sub-priority & 0.50 & 0.73 & $\mathbf{0 . 8 4}$ \\
Sub-role & 0.53 & 0.83 & $\mathbf{0 . 9 6}$ \\
\hline Micro / Macro average & - & $0.75 / 0.68$ & $\mathbf{0 . 9 0} / \mathbf{0 . 9 0}$ \\
\hline
\end{tabular}


Table 1 shows the evaluation scores of $\mathrm{REaCT}^{2}$ compared to the results of previous versions based on the original dataset of Dollmann and Geierhos (2016: 1809ff.). It is clear that we improved the overall result significantly. This especially applies to the semantic categories of "Refinement of component" (0.95), "Sub-action" (0.93) and "Argument of sub-action" (0.85). But where there is light, there is also shadow: For example, we need to improve our tool in the categories "Condition" (-0.16) and "Component" (-0.05).

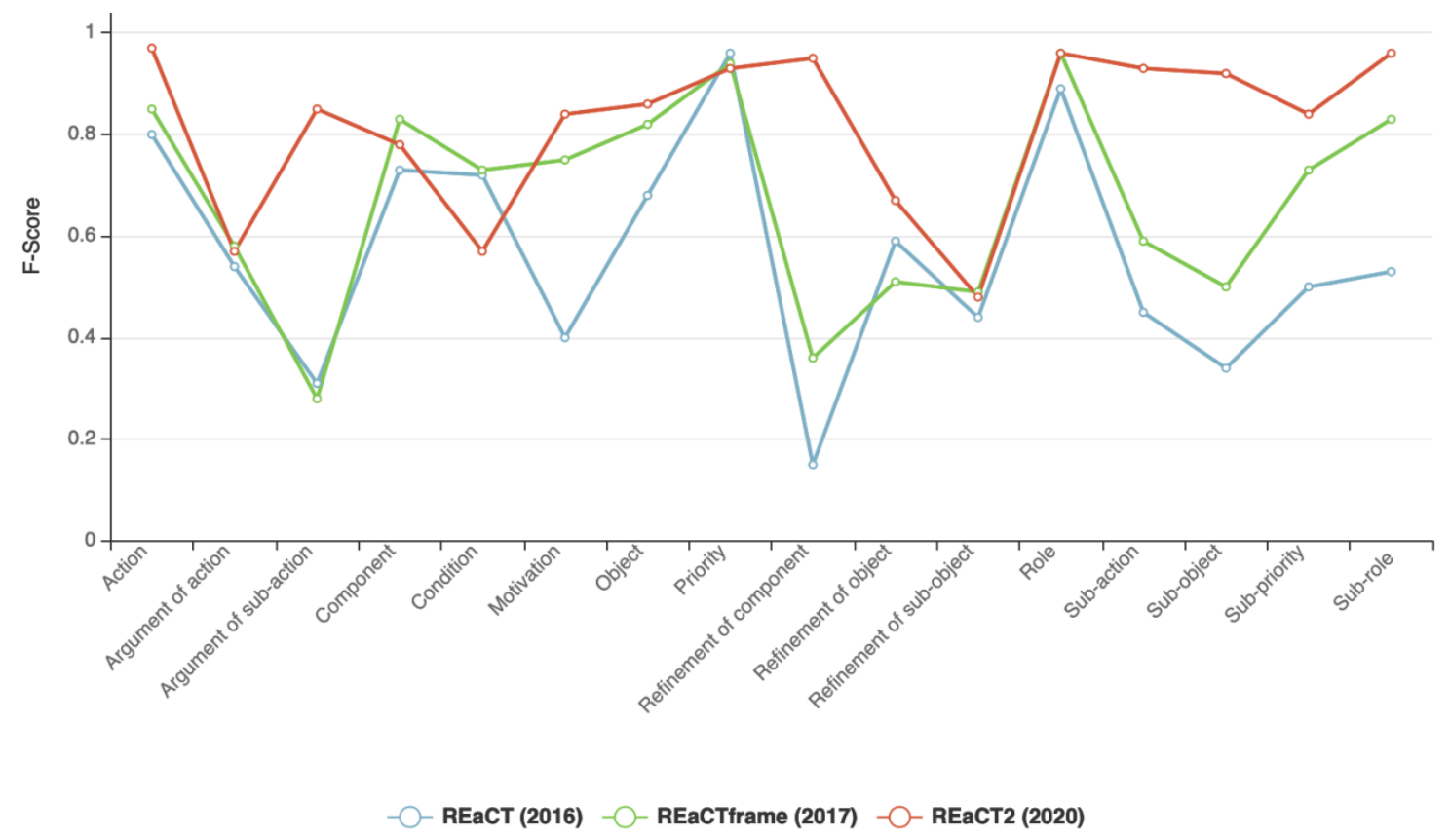

Figure 2. REaCT and REaCT frame vs. the proposed model (F1 scores)

However, we are very optimistic, since we have not yet exhausted all possibilities of model optimization (ongoing work) and, due to the increased use of the prototypes, we will have a larger dataset for the fine-tuning of the language models available over time. In contrast to the training data, there is no need to annotate it and we define it as part of the OTF Computing maintenance workflow. REaCT ${ }^{2}$ seems to be a good contribution to the OTF Computing use case as well as to the user satisfaction and we will continue to work on it.

\section{CONCLUSION}

NL software requirements are an important tool when it comes to integrating the requirements of end users into a software product, especially of those who have no technical knowledge. In OTF Computing, this situation is intensified as the intermediate level of developers and software project managers is eliminated and end users contribute directly to a software service through their software requirements.

The state of research is small when it comes to extracting requirements from unstructured texts and decomposing them on a semantic level. It is small because it is very difficult to obtain real training data. In this short paper we show that the problem of scarce resources can be satisfactorily bypassed, since the latest achievements in the field of ML methods and NLP make it possible to further develop existing language models. Following this approach, the previous works $\mathrm{REaCT}$ and $\mathrm{REaCT}_{\text {frame }}$ are outperformed.

We will continue this approach in the future research, because with our software tool CORDULA2 (Bäumer 2019: 10ff.) we also follow interactive approaches using chatbots, which allow users to fix even errors such as ambiguities in their requirements (Bäumer 2019: 4). Here, transformer-based models can also help to improve the communication between the software system and end user. 


\section{ACKNOWLEDGEMENT}

Special thanks to Markus Dollmann and Michaela Geierhos, the original authors of REaCT, for their support and sharing training data for comparative benchmarks. This work was partially supported by the German Research Foundation (DFG) within the Collaborative Research Centre "On-The-Fly Computing” (SFB 901).

\section{REFERENCES}

Bäumer, F., et al, 2019. Natural Language Processing in OTF Computing: Challenges and the Need for Interactive Approaches. Computers, Vol. 8, No. 122, pp. 1-14.

Caron, M. et al, 2018. Back to Basics: Extracting Software Requirements with a Syntactic Approach. Joint Proceedings of REFSQ-2018 Workshops, Doctoral Symposium, Live Studies Track, and Poster Track co-located with the 23rd International Conference on Requirements Engineering: Foundation for Software Quality. Utrecht, NL, Vol. 2075.

Devlin, J., et al, 2018. BERT: Pre-training of Deep Bidirectional Transformers for Language Understanding. CoRR. 1810.04805, cs.CL.

Dollmann, M., and Geierhos, M., 2016. On- and Off-Topic Classification and Semantic Annotation of User-Generated Software Requirements. Proceedings of the 2016 Conference on Empirical Methods in Natural Language Processing (EMNLP). Austin, TX, USA, pp. 1807-1816. Association for Computational Linguistics (ACL).

Karl, H., et al, 2019. A Case for a New IT Ecosystem: On-The-Fly Computing. Business \& Information Systems Engineering, pp. 1-13.

Kim, Y. et al, 2017. Semantic Annotation of Software Requirements with Language Frame. International Journal of Software Engineering for Smart Device, Vol. 4, No. 2, pp. 1-6.

Kim, Y., et al, 2018. Improving Classifiers for Semantic Annotation of Software Requirements with Elaborate Syntactic Structure. International Journal of Advanced Science and Technology, Vol. 112, pp.123-136.

Lewis, Mike, et al, 2019. BART: Denoising Sequence-to-Sequence Pre-training for Natural Language Generation, Translation, and Comprehension. CoRR. 1910.13461v1, cs.CL.

Liu, Y., et al, 2019. RoBERTa: A Robustly Optimized BERT Pretraining Approach. CoRR. 1907.11692, cs.CL.

Peters, M., et al, 2018. Deep Contextualized Word Representations. Proceedings of the 2018 Conference of the North American Chapter of the ACL: Human Language Technologies, pp. 2227-2237.

Radford, A., et al, 2018. Improving Language Understanding by Generative Pre-Training. Technical Report, pp. 1-12.

Rajapakse, T., 2020. Transformers for Classification, NER, QA, Language Modelling, Language Generation, T5, Multi-Modal, and Conversational AI. Web Resource: https://github.com/ThilinaRajapakse/simpletransformers, 2020-07-09

Vaswani, A., et al, 2017. Attention is All You Need. Proceedings of the 31st Conference on Neural Information Processing Systems, pp. 5998-6008. 